\title{
The levels of inflammatory biomarkers in hemodialysis and peritoneal dialysis patients
}

\author{
Mehmet Yildirim* (), Seyyid Bilal Acikgoz ${ }^{2}(\mathbb{0})$, Ahmed Bilal Genc ${ }^{3}(\mathbb{B}$, \\ Selçuk Yaylaci ${ }^{3}$, Hamad Dheir ${ }^{4}$, , Savaş Sipahi ${ }^{4}$
}

\section{SUMMARY}

OBJECTIVE: In this study, we aimed to determine fibroblast growth factor 23, soluble alpha klotho, osteocalcin, indoxyl sulphate, sclerostin, Procollagen $1 \mathrm{~N}$ Terminal Propeptide, and beta-CrossLaps levels in hemodialysis and peritoneal dialysis patients, and to compare the levels of these markers among hemodialysis and peritoneal dialysis patients, as well as healthy individuals.

METHODS: The study included 30 hemodialysis and 23 peritoneal dialysis patients who were followed-up for at least six months at the Sakarya University Hospital, besides 30 healthy volunteers.

RESULTS: The participants were divided into three groups with similar characteristics in terms of age, gender and body mass index. Fibroblast growth factor 23, soluble alpha klotho, indoxyl sulphate, beta-CrossLaps, and Procollagen $1 \mathrm{~N}$ Terminal Propeptide levels were significantly higher in patients of both the hemodialysis and peritoneal dialysis groups than in the healthy volunteers' group. There was no difference in levels of these molecules between hemodialysis and peritoneal dialysis groups.

CONCLUSIONS: Fibroblast growth factor 23, sclerostin, indoxyl sulphate, beta-CrossLaps, and Paclitaxel-induced neuropathic pain levels were higher in patients of both groups as inflammatory markers. In our study, we found higher soluble alpha klotho levels in patients of both groups than in the healthy volunteers' group, suggesting that blood soluble alpha klotho levels may not correlate with renal klotho levels. KEYWORDS: Kidney disease, chronic. End-stage renal disease. Inflammatory markers.

\section{INTRODUCTION}

Chronic kidney disease (CKD) has become an important health problem worldwide causing progressive illness, bone-mineral disorders, cardiovascular morbidities, and early deaths. In the course of CKD, bone-mineral disorders may occur due to the high level of blood phosphorus. Hyperphosphatemia causes secondary hyperparathyroidism, decreasing blood calcium and calcitriol levels. This clinical condition is defined as renal osteodystrophy (ROD). It may cause cardiovascular diseases, pathological bone fracture and, finally, increased risk of mortality ${ }^{1}$.
Although the process pathophysiology has not been well understood yet, vascular calcification has been an initiator of vascular inflammation ${ }^{2}$. Therefore, the biomarkers, which show vascular inflammation in the course of CKD, could provide clinicians with early diagnosis and treatment of the CKD complications. In this piece of article, we will focus on inflammation pathways and potential inflammatory biomarkers.

As mentioned, secondary hyperparathyroidism starts the process of ROD. Loss of nephrons may cause phosphate retention in circulation and bones respond to it with increased

\footnotetext{
${ }^{1}$ Hacettepe University Faculty of Medicine, Intensive Care Unit, Internal Medicine Department - Ankara, Turkey.

${ }^{2}$ Samsun Ondokuz Mayıs University Faculty of Medicine, Internal Medicine-Rheumotology Department - Samsun, Turkey.

${ }^{3}$ Sakarya University School of Medicine, Internal Medicine Department - Sakarya-Turkey.

${ }^{4}$ Sakarya University School of Medicine, Nephrology Department - Sakarya, Turkey.

*Corresponding author: dryildirimmehmet@hotmail.com, drseyyid@gmail.com

Conflicts of interest: the authors declare there is no conflicts of interest. Funding: this study was funded by Sakarya University Scientific Research

Project Projects Unit (project number: 2017-40-02-002).

Received on March 14, 2021. Accepted on March 21, 2021.
} 
fibroblast growth factor-23 (FGF-23) production. Excreted phosphorus levels increase in urine and FGF-23 binds both the FGF-23 receptors and the klotho. Additionally, FGF-23 inhibits cytochrome 27B1 (CYP-27B1) enzyme, which converts 25 hydroxyvitamin $\mathrm{D}$ to $1-25$ dihydroxyvitamin $\mathrm{D}$ and increases renal calcium reabsorption using the transient receptor potential vanilloid 5 (TRPV-5) channel that needs klotho for activation. Studies have shown that FGF-23 concentration increases in patients with stage- 2 and upper $\mathrm{CKD}$, and may reach 1,000 times of normal range ${ }^{3,4}$. A previous study, in a large cohort, has reported that increased FGF-23 levels are independently associated with mortality among patients who are beginning hemodialysis treatment, suggesting further investigation to reveal whether FGF-23 might be a new biomarker ${ }^{5}$.

Klotho is a protein secreted from multiple tissues, especially kidneys. Klotho has antiapoptotic, antioxidant, angiogenetic and antifibrinogenetic effects. In addition, klotho suppresses phosphate reabsorption and activates phosphate excretion and appears to protect kidneys in patients with CKD. In these individuals, soluble alpha klotho levels decrease due to impaired renal functions. Studies have shown that klotho may be a predictor of early disease and klotho deficiency may be an indicator of disease progression and complication, such as bone disorders and vascular inflammation ${ }^{6}$.

Indoxyl sulphate is the uremic toxin produced by tryptophan metabolism and secreted from proximal tubules. In patients with CKD, secretion of indoxyl sulphate decreases. It has been proven that high levels of indoxyl sulphate are associated with endothelial oxidative stress, atherosclerosis and vascular inflammation? ${ }^{7}$.

Osteocalcin is an osteoblast-specific protein secreted from bones that regulates phosphorus and vitamin $\mathrm{D}$ metabolism and sexual functions. On one hand, it is expected that in the course of $\mathrm{CKD}$, osteocalcin levels increase as a result of bone resorption. On the other hand, a study has reported a negative relationship between osteocalcin levels and mortality in patients with coronary artery disease ${ }^{8}$.

Sclerostin is a glycopeptide secreted from osteocytes. Sclerostin inhibits Wnt pathway, which plays an anabolic role in bone metabolism. There is a negative correlation between blood sclerostin and parathyroid hormone (PTH) levels in hemodialysis patients ${ }^{9}$. A recent study has reported that higher sclerostin levels may prolong life expectancy in hemodialysis patients ${ }^{10}$.

Procollagen 1 N Terminal Propeptide (PINP) is a bone formation marker produced during collagen synthesis. Osteoclasts, acids and neutral proteases reveal fragments containing C-terminal telopeptide in the process of bone destruction. Aspartic acid is added to these telopeptides and then beta-CrossLaps are formed. Beta-CrossLaps are released into the circulation, and when they are detected in the blood, it may result in the bone resorption and mature type 1 collagen degradation. Beta-CrossLaps levels may increase up to five times in patients undergoing hemodialysis compared with healthy volunteers ${ }^{11}$.

Vascular inflammation and bone disorders are life-threatening complications of CKD and have been associated with severe complications in patients with CKD. These complications should be treated in the early phases of disease to improve prognosis. Therefore, markers that show this process to the clinicians in the early phases of these complications are strongly needed. FGF23, klotho, indoxyl sulphate, osteocalcin, sclerostin, PINP and beta-CrossLaps are the markers that have recently been focused on. However, there has been no consensus on the routine used for these molecules yet, because their normal and risky levels have not been evaluated clearly yet and there are very limited data about the levels of these molecules in patients who undergo peritoneal dialysis.

The primary aim of this study was to compare the levels of these markers in dialysis patients and healthy population. Secondly, we aimed to identify the relationship between type of renal replacement therapy (RRT) in hemodialysis and peritoneal dialysis and the level of these markers.

\section{METHODS}

\section{Patient selection}

We have conducted a prospective cohort study including patients with CKD, who received hemodialysis and peritoneal dialysis treatments, and healthy volunteers. A total of 30 hemodialysis and 23 peritoneal dialysis patients, besides 30 healthy volunteers were included. Both groups of patients consisted of patients with CKD that had been receiving dialysis treatment for at least six months. The age range of patients was 18-80 years. Exclusion criteria were: temporary renal dysfunction, active infectious/ inflammatory episodes or acute ischaemic vascular disease histories during the previous three months, additional diseases causing chronic inflammation, chronic liver disease and positive hepatitis serology. Additionally, patients who did not provide written consent were excluded from the study. Healthy volunteers in the age range of 18-80 years, who did not have chronic diseases and infectious diseases during the last three months were included in the study. All volunteers provided written consent.

\section{Data collection}

Demographic data (e.g. gender, age, start date of dialysis treatment, comorbidities, systolic and diastolic blood pressure and body mass index) and laboratory data (creatinine, C-Reactive Protein - CRP, albumin - A, calcium - Ca, phosphorus - P, Parathyroid hormone - PTH, total cholesterol, low-density lipoprotein $-\mathrm{LDL}$, haemoglobin $-\mathrm{Hb}$ ) of both groups of patients 
were analyzed. In order to determine the levels of inflammatory biomarkers (FGF-23, soluble alpha klotho, indoxyl sulphate, osteocalcin, sclerostin, PINP, beta-CrossLaps), the blood samples of hemodialysis patients were collected before the second hemodialysis during a mid-week session, and the blood samples of peritoneal dialysis patients were collected before morning changes. These blood samples were stored at $-80^{\circ} \mathrm{C}$. Human FGF-23, sclerostin, osteocalcin, indoxyl sulfate, Procollagen $1 \mathrm{~N}$ Terminal Propeptide, soluble alpha klotho and Beta-CrossLaps ELISA kits were used to determine the molecular levels (supplied by Hangzhou Eastbiopharm Co., Ltd. - PRC, China). Due to the manufacturer instructions, $40 \mathrm{U} / \mathrm{L}$ of blood sample were collected from each volunteer and tested with microELISA method. Results were identified with Triturus (Grifols) ELISA instrument with a 450-nm wavelength.

\section{Statistical analysis}

The Statistical Package for the Social Sciences (SPSS) version 22.0 was used for all statistical analyses. Data were shown, such as frequency (percentage), number and meantstandard deviation. Kolmogorov-Smirnov test was used for assessing the normality of the distribution of the numerical variables. Student's- $t$ and ANOVA tests were used to compare the differences among the groups regarding normally distributed numerical variables. Mann-Whitney U and Kruskal Wallis tests were applied to compare the differences among the groups regarding non-normally distributed variables. $\chi^{2}$ test was used to assess categorical variables. Spearman's correlation analysis determined the relationship among non-normally distributed variables. Level of significance was accepted as $\mathrm{p}<0.05$.

\section{RESULTS}

Demographic data and baseline characteristics of each group are shown in Table 1 . There was no statistically significant difference for age, gender and body mass index among the three groups $(\mathrm{p}=0.743 ; \mathrm{p}=0.421 ; \mathrm{p}=0.381$, respectively). In hemodialysis group, patients underwent dialysis treatment for $75.13 \pm 43.18$ months; and in peritoneal dialysis group, $42.13 \pm 29.46$ months $(\mathrm{p}<0.05)$. Eight $(26.7 \%)$ patients of hemodialysis group and $6(26.1 \%)$ patients of peritoneal dialysis group had a diabetes history, which did not show any significant difference. In both

Table 1. Demographic data, baseline characteristics and laboratory findings.

\begin{tabular}{|c|c|c|c|c|}
\hline & Hemodialysis & Peritoneal dialysis & Healthy volunteers & \multirow{2}{*}{ p-value } \\
\hline & $(n=30)$ & $(n=23)$ & $(n=30)$ & \\
\hline Age, years & $52.50 \pm 16.03$ & $52.35 \pm 14.15$ & $50.07 \pm 15.51$ & $0.743^{*}$ \\
\hline Gender, F/M (\%) & $20(66.7) / 10(3.3)$ & $13(56.5) / 10(43.5)$ & $15(50) / 15(50)$ & $0.421^{\dagger}$ \\
\hline Dialysis period (months) & $75.13 \pm 43.18$ & $42.13 \pm 29.46$ & - & $0.003^{\ddagger}$ \\
\hline Diabetes n (\%) & $8(26.7)$ & $6(26.1)$ & - & $0.962^{+}$ \\
\hline Systolic blood pressure $(\mathrm{mmHg})$ & $140.59 \pm 27.04$ & $129.45 \pm 27.57$ & $117.47 \pm 17.65$ & $0.002^{*}$ \\
\hline Diastolic blood pressure $(\mathrm{mmHg})$ & $89.56 \pm 23.01$ & $85.77 \pm 19.88$ & $76.50 \pm 15.52$ & $0.040^{*}$ \\
\hline BMI & $24.23 \pm 4.32$ & $25.38 \pm 5.08$ & $25.86 \pm 4.38$ & $0.381 *$ \\
\hline Creatinine $(\mathrm{mg} / \mathrm{dL})$ & $9.94 \pm 2.30$ & $8.51 \pm 3.00$ & $0.86 \pm 0.22$ & $<0.001 *$ \\
\hline $\mathrm{CRP}(\mathrm{mg} / \mathrm{L})$ & $12.67 \pm 12.55$ & $28.61 \pm 44.68$ & $5.37 \pm 5.57$ & $0.004^{\S}$ \\
\hline Albumin (mg/dL) & $4.03 \pm 0.30$ & $3.19 \pm 0.57$ & $4.10 \pm 0.23$ & $<0.001^{\S}$ \\
\hline $\mathrm{Ca}(\mathrm{mg} / \mathrm{dL})$ & $8.56 \pm 0.69$ & $9.16 \pm 0.94$ & $9.40 \pm 0.28$ & $<0.001 *$ \\
\hline$P(\mathrm{mg} / \mathrm{dL})$ & $5.44 \pm 1.16$ & $5.34 \pm 1.59$ & $2.99 \pm 0.59$ & $<0.001 *$ \\
\hline CaxP & $46.48 \pm 9.78$ & $48.42 \pm 13.37$ & $28.30 \pm 6.10$ & $<0.001 *$ \\
\hline PTH $(p g / m L)$ & $804.19 \pm 741.25$ & $461.81 \pm 332.20$ & $60.48 \pm 16.46$ & $<0.001^{\S}$ \\
\hline Total cholesterol (mg/dL) & $163.97 \pm 35.81$ & $204.78 \pm 62.84$ & $219.08 \pm 41.12$ & $<0.001^{\S}$ \\
\hline $\mathrm{LDL}(\mathrm{mg} / \mathrm{dL})$ & $97.63 \pm 30.95$ & $124.30 \pm 43.79$ & $156.04 \pm 34.42$ & $<0.001^{\S}$ \\
\hline Haemoglobin (g/dL) & $11.08 \pm 1.47$ & $10.33 \pm 1.40$ & $13.65 \pm 1.57$ & $<0.001 *$ \\
\hline
\end{tabular}

F: feminine; M: masculine; BMI: body mass index; CRP: C-Reactive Protein; Ca: - Calcium; P: phosphorus; CaxP: Calcium phosphate product; PTH: parathyroid hormone; LDL: low density lipoprotein.

*One-Way ANOVA Test; ${ }^{\dagger}$ Ki-Kare Test; ${ }^{*}$ Mann Whitney U Test; ${ }^{\circledR}$ Kruskal Wallis Test 
groups of patients, systolic $(\mathrm{p}=0.002)$ and diastolic blood pressure $(\mathrm{p}=0.04)$ were higher than in the healthy volunteers' group. The mean CRP levels of hemodialysis and peritoneal dialysis patients were higher than in healthy volunteers $(12.67 \pm 12.55$; $28.61 \pm 44.68 ; 5.37 \pm 5.57 \mathrm{mg} / \mathrm{L}$, respectively, $\mathrm{p}=0.004)$. The mean albumin levels of healthy volunteers $(4.10 \pm 0.23 \mathrm{mg} / \mathrm{dL})$ were higher than in hemodialysis $(4.03 \pm 0.30 \mathrm{mg} / \mathrm{dL})$ and peritoneal dialysis patients $(3.19 \pm 0.57 \mathrm{mg} / \mathrm{dL} ; \mathrm{p}<0.001)$. The mean calcium, phosphorus and PTH levels of hemodialysis and peritoneal dialysis patients were higher than in healthy volunteers $(\mathrm{p}<0.001 ; \mathrm{p}<0.001 ; \mathrm{p}<0.001)$. The mean of total cholesterol and LDL levels were higher in patients of both groups than in the healthy volunteers' group $(\mathrm{p}<0.001 ; \mathrm{p}<0.001)$.

The mean blood levels of FGF-23, soluble alpha klotho, osteocalcin, indoxyl sulphate, sclerostin, PINP and beta-CrossLaps are listed in Table 2. The mean blood levels of FGF-23, soluble alpha klotho, indoxyl sulphate, sclerostin and PINP were significantly higher in hemodialysis and peritoneal dialysis groups than in the healthy volunteers' group $(\mathrm{p}<0.001)$, while there was no significant difference among these groups in terms of mean blood levels of osteocalcin $(\mathrm{p}=0.134)$. We found a positive correlation between soluble alpha klotho levels and systolic blood pressure in hemodialysis group $(\mathrm{p}=0.039$; $\mathrm{r}=0.399$ ). In the peritoneal dialysis group, there was an inverse correlation between soluble alpha klotho and CRP levels $(\mathrm{p}=0.008 ; \mathrm{r}=-0.539)$.

\section{DISCUSSION}

FGF-23, soluble alpha klotho, indoxyl sulphate, sclerostin, PINP and beta-CrossLaps levels were significantly higher in both groups of patients than in the healthy volunteers' group, which is consistent with previous studies ${ }^{12}$.

Studies showed a strong correlation between serum creatinine and FGF-23 concentration ${ }^{4,13}$. In our study, FGF-23 levels in hemodialysis patients were higher and they were also determined in a wide range. Lima et al. compared FGF-23 levels and bone histomorphometry parameters of dialysis patients and revealed that circulating FGF-23 concentrations may indicate alterations in ongoing bone formation ${ }^{14}$. Therefore, our study supports previous researches that found FGF-23 levels in a wide range $\mathrm{e}^{13,14}$. It appears that a large meta-analysis is needed to determine the range of FGF-23 levels for CKD.

In the course of $\mathrm{CKD}$, decrease of renal klotho expression causes increase of FGF-23 concentration. Firstly, Shimamura et al. reported that low levels of soluble alpha klotho could be a new marker in $\mathrm{CKD}^{15}$. However; some recent studies have shown that soluble alpha klotho levels may not reflect tissue klotho expression ${ }^{16}$. Seiler et al. followed up 312 patients with CKD for 2.2 years and found out that the level of soluble alpha klotho was not associated with severity of renal dysfunction and complications ${ }^{17}$. Interestingly, in the current study, soluble alpha klotho levels have been significantly higher in both groups of patients than in the healthy volunteers' group. Therefore, we can speculate that soluble alpha klotho levels in patients on dialysis treatment may not be a reliable marker or may not reflect the expression of renal klotho directly.

In 2011, a study indicated a negative correlation between glomerular filtration rate and indoxyl sulphate levels ${ }^{18}$. Furthermore, a meta-analysis confirmed the positive relationship between indoxyl sulphate and mortality ${ }^{19}$. As expected, in our study, the indoxyl sulphate levels were significantly higher in both groups of patients, and there was no statistically significant difference between hemodialysis and peritoneal dialysis groups.

Beta-CrossLaps and PINP have been used as bone loop markers in volunteers that do not have CKD. It has not been clear yet whether beta-CrossLaps and PINP can be used as bone turnover markers ${ }^{20}$. Nevertheless, few previous studies reported that beta-CrossLaps levels were significantly higher in patients undergoing hemodialysis ${ }^{21,22}$. Although there are limited studies

Table 2. Blood levels of markers.

\begin{tabular}{l|c|c|c|c} 
& $\begin{array}{c}\text { Hemodialysis } \\
(\mathrm{n}=30)\end{array}$ & $\begin{array}{c}\text { Peritoneal dialysis } \\
(\mathrm{n}=23)\end{array}$ & $\begin{array}{c}\text { Healthy volunteers } \\
(\mathrm{n}=30)\end{array}$ & p-value \\
\hline FGF-23 $(\mathrm{pg} / \mathrm{mL})$ & $772.33 \pm 369.74$ & $657.13 \pm 339.30$ & $325.40 \pm 232.44$ & $<0.001^{*}$ \\
\hline Soluble alpha klotho $(\mathrm{ng} / \mathrm{mL})$ & $12.06 \pm 3.72$ & $11.65 \pm 4.42$ & $6.79 \pm 3.02$ & $<0.001^{\dagger}$ \\
\hline Indoxyl sulphate $(\mathrm{mcg} / \mathrm{mL})$ & $51.09 \pm 13.18$ & $47.83 \pm 14.96$ & $21.52 \pm 5.55$ & $<0.001^{*}$ \\
\hline Osteocalcin $(\mathrm{ng} / \mathrm{mL})$ & $61.38 \pm 31.12$ & $52.90 \pm 19.52$ & $46.89 \pm 28.47$ & $0.134^{*}$ \\
\hline Sclerostin $(\mathrm{ng} / \mathrm{mL})$ & $1.073 \pm 0.590$ & $0.825 \pm 0.445$ & $0.452 \pm 0.219$ & $<0.001^{*}$ \\
\hline PINP $(\mathrm{ng} / \mathrm{mL})$ & $1113.03 \pm 511.10$ & $946.96 \pm 528.03$ & $431.37 \pm 254.16$ & $<0.001^{*}$ \\
\hline Beta-CrossLaps $(\mathrm{ng} / \mathrm{L})$ & $1486.90 \pm 821.48$ & $1163.35 \pm 627.86$ & $680.83 \pm 508.52$ & $<0.001^{*}$ \\
\hline
\end{tabular}

*Kruskal-Wallis Test; ${ }^{\dagger}$ One-way ANOVA Test 
focusing on the levels of these markers among peritoneal dialysis patients, a recent study has found out increased levels of beta-CrossLaps in peritoneal dialysis patients. However, hemodialysis patients have not been included in this previous study ${ }^{23}$. Our study has demonstrated that in dialysis patients beta-CrossLaps levels have been significantly higher than in healthy volunteers and there have been no statistically significant differences between hemodialysis and peritoneal dialysis patients.

In 2015, Liu and He reported that patients who received dialysis had significantly higher PINP levels than those who did not receive dialysis ${ }^{24}$. In the current study, PINP, which is a marker of bone formation, and the precursor molecule of beta-CrossLaps have been considered higher in both groups of patients than in the healthy volunteers' group.

A previous study from Austria has detected two times higher sclerostin levels in dialysis patients than in healthy volunteer ${ }^{25}$. In line with, the present study has shown that sclerostin levels were significantly higher in both groups of patients than in the healthy volunteers' group and there was no statistically significant difference between hemodialysis and peritoneal dialysis patients. However, another study has shown an inverse correlation between sclerostin and glomerular filtration rate in patients with CKD and those who did not receive dialysis treatment ${ }^{12}$. Further studies comparing sclerostin levels of pre-dialysis and dialysis patients are needed to enlighten these findings.

In the current study, we have not found differences in the osteocalcin levels between patients and healthy volunteers. It has been a remarkable finding, because there was no similar finding in the previous researches. Two studies ( $\mathrm{n}=61$ and $\mathrm{n}=98$ ) have reported an inverse correlation between glomerular filtration rate and osteocalcin levels ${ }^{26,27}$. Also, it can be related to the fact that there have been fewer patients in our study than in the literature. Researches in larger cohorts are needed to confirm our findings.

There have been some limitations in the current study. Firstly, the longer treatment duration of the hemodialysis group might have caused higher levels of inflammatory biomarkers in this group. Secondly, there has been no data on patients' long period follow-up that could suggest the relationship between levels of markers and severe clinical complications of CKD.
Finally, we could not determine the effects of patients' medications on biomarker levels.

\section{CONCLUSIONS}

In the present study, FGF-23, indoxyl sulphate, sclerostin, beta-CrossLaps and PINP levels have been significantly higher in both hemodialysis and peritoneal dialysis group than in the healthy volunteers' group. There has been no significant difference of these markers between hemodialysis and peritoneal dialysis patients. Soluble alpha klotho levels in dialysis patients have been higher than in healthy volunteers, speculating that blood soluble alpha klotho levels may not directly reflect the renal klotho expression.

\section{ACKNOWLEDGEMENTS}

Ethics committee approval of this study was obtained from Sakarya University, School of Medicine Ethics Committee, in document number 16214662/050.01.04/150.

This study was funded by Sakarya University Scientific Research Projects Unit (project number: 2017-40-02-002).

\section{AUTHORS" CONTRIBUTIONS}

MY: Conceptualization, Data curation, Formal analysis, Investigation, Methodology, Project administration, Supervision, Validation, Visualization, Writing - original draft, Writing - review and editing. SBA: Data curation, Formal analysis, Investigation, Methodology, Project administration, Resources, Software, Supervision, Validation. ABG: Data curation, Formal analysis, Investigation, Methodology, Project administration, Resources, Software, Supervision, Writing - review and editing. SY: Conceptualization, Data curation, Formal analysis, Investigation, Methodology, Writing - review and editing. HD: Conceptualization, Investigation, Methodology, Writing - original draft, Writing - review and editing. SS: Conceptualization, Formal analysis, Investigation, Methodology, Project administration, Resources, Supervision, Writing - original draft, Writing - review and editing.

\section{REFERENCES}

1. Go AS, Chertow GM, Fan D, McCulloch CE, Hsu C. Chronic kidney disease and the risks of death, cardiovascular events, and hospitalization. N Engl J Med. 2004;351(13):1296-305. https://doi.org/10.1056/NEJMoa041031

2. London GM, Marchais SJ, Guérin AP, Métivier F. Arteriosclerosis, vascular calcifications and cardiovascular disease in uremia. Curr Opin Nephrol Hypertens.
2005;14(6):525-31. https://doi.org/10.1097/01 mnh.0000168336.67499.c0

3. Shimada T, Urakawa I, Isakova T, Yamazaki Y, Epstein M, Wesseling-Perry K, et al. Circulating fibroblast growth factor 23 in patients with end-stage renal disease treated by peritoneal dialysis is intact and biologically active. J Clin Endocrinol Metab. 2010;95(2):578-85. https://doi.org/10.1210/jc.2009-1603 
4. Isakova T, Wahl P, Vargas GS, Gutiérrez OM, Scialla J, Xie H, et al. Fibroblast growth factor 23 is elevated before parathyroid hormone and phosphate in chronic kidney disease. Kidney Int. 2011;79(12):1370-8. https://doi.org/10.1038/ki.2011.47

5. Gutiérrez OM, Mannstadt M, Isakova T, Rauh-Hain JA, Tamez $\mathrm{H}$, Shah A, et al. Fibroblast growth factor 23 and mortality among patients undergoing hemodialysis. N Engl J Med. 2008;359(6):584-92. https://doi.org/10.1056/NEJMoa0706130

6. Hu MC, Kuro-o M, Moe OW. The emerging role of Klotho in clinical nephrology. Nephrol Dial Transplant. 2012;27(7):2650-7. https://doi.org/10.1093/ndt/gfs160

7. Yamamoto $\mathrm{H}$, Tsuruoka $\mathrm{S}$, loka $\mathrm{T}$, Ando $\mathrm{H}$, Ito $\mathrm{C}$, Akimoto $\mathrm{T}$, et al. Indoxyl sulfate stimulates proliferation of rat vascular smooth muscle cells. Kidney Int. 2006;69(10):1780-5. https:// doi.org/10.1038/sj.ki.5000340

8. Parker BD, Schurgers LJ, Brandenburg VM, Christenson RH, Vermeer $C$, Ketteler $M$, et al. The associations of fibroblast growth factor 23 and uncarboxylated matrix Gla protein with mortality in coronary artery disease: the Heart and Soul Study. Ann Intern Med. 2010;152(10):640-8. https://doi. org/10.7326/0003-4819-152-10-201005180-00004

9. Cejka D, Herberth J, Branscum AJ, Fardo DW, Monier-Faugere MC, Diarra D, et al. Sclerostin and Dickkopf-1 in renal osteodystrophy. Clin J Am Soc Nephrol. 2011;6(4):877-82. https://doi.org/10.2215/CJN.06550810

10. Gonçalves FLC, Elias RM, dos Reis LM, Graciolli FG, Zampieri FG, Oliveira RB, et al. Serum sclerostin is an independent predictor of mortality in hemodialysis patients. BMC Nephrol. 2014;15;190. https://doi.org/10.1186/1471-2369-15-190

11. Reichel $H$, Roth HJ, Schmidt-Gayk H. Evaluation of serum beta-carboxy-terminal cross-linking telopeptide of type I collagen as marker of bone resorption in chronic hemodialysis patients. Nephron Clin Pract. 2004;98(4):c112-8. https://doi. org/10.1159/000081552

12. Pelletier S, Dubourg L, Carlier MC, Hadj-Aissa A, Fouque D. The relation between renal function and serum sclerostin in adult patients with CKD. Clin J Am Soc Nephrol. 2013;8(5):819-23. https://doi.org/10.2215/CJN.07670712

13. Larsson $T$, Nisbeth $U$, Ljunggren $O$, Jüppner $H$, Jonsson $K B$. Circulating concentration of FGF-23 increases as renal function declines in patients with chronic kidney disease, but does not change in response to variation in phosphate intake in healthy volunteers. Kidney Int. 2003;64(6):2272-9. https:// doi.org/10.1046/j.1523-1755.2003.00328.x

14. Lima F, El-Husseini A, Monier-Faugere M, David V, Mawad $H$, Quarles D, et al. FGF-23 serum levels and bone histomorphometric results in adult patients with chronic kidney disease on dialysis. Clin Nephrol. 2014;82(5):287-95. https://doi.org/10.5414/CN108407

15. Shimamura $Y$, Hamada K, Inoue K, Ogata K, Ishihara M, Kagawa T, et al. Serum levels of soluble secreted $\alpha$-Klotho are decreased in the early stages of chronic kidney disease, making it a probable novel biomarker for early diagnosis. Clin Exp Nephrol. 2012;16(5):722-9. https://doi.org/10.1007/ s10157-012-0621-7
16. Drüeke TB, Massy ZA. Circulating Klotho levels: clinical relevance and relationship with tissue Klotho expression. Kidney Int. 2013;83(1):13-5. https://doi.org/10.1038/ki.2012.370

17. Seiler S, Wen M, Roth HJ, Fehrenz M, Flügge $F$, Herath $E$, et al. Plasma Klotho is not related to kidney function and does not predict adverse outcome in patients with chronic kidney disease. Kidney Int. 2013;8(1):121-8. https://doi.org/10.1038/ ki.2012.288

18. Wu IW, Hsu KH, Lee CC, Sun CY, Hsu HJ, Tsai CJ, et al. p-Cresyl sulphate and indoxyl sulphate predict progression of chronic kidney disease. Nephrol Dial Transplant. 2011;26(3):938-47. https://doi.org/10.1093/ndt/gfq580

19. Lin CJ, Wu V, Wu PC, Wu CJ. Meta-analysis of the associations of p-cresyl sulfate (PCS) and indoxyl sulfate (IS) with cardiovascular events and all-cause mortality in patients with chronic renal failure. PLoS One. 2015;10(7):e0132589. https://doi. org/10.1371/journal.pone.0132589

20. Delanaye P, Souberbielle JC, Lafage-Proust MH, Jean G, Cavalier E. Can we use circulating biomarkers to monitor bone turnover in CKD haemodialysis patients? Hypotheses and facts. Nephrol Dial Transplant. 2014;29(5);997-1004. https://doi.org/10.1093/ndt/gft275

21. Okabe R, Nakatsuka K, Inaba M, Miki T, Naka H, Masaki H, et al. Clinical evaluation of the Elecsys beta-CrossLaps serum assay, a new assay for degradation products of type I collagen C-tlopeptides. Clin Chem. 2001;47(8):1410-4. PMID: 11468230

22. Cavalier E, Delanaye P, Collette J, Krzesinski JM, Chapelle JP. Evaluation of different bone markers in hemodialyzed patients. Clin Chim Acta. 2006;371(1-2):107-11. https://doi. org/10.1016/j.cca.2006.02.029

23. Yilmaz VT, Ozdem S, Donmez L, Cetinkaya R, Suleymanlat G, Ersoy FF. FGF-23, $\alpha$-Klotho gene polymorphism and their relationship with the markers of bone metabolism in chronic peritoneal dialysis patients. Eurasian J Med. 2015;47(2):11525. https://doi.org/10.5152/eurasianjmed.2015.93

24. Liu Y, He Q. Alkaline phosphatase, iPTH and bone turnover markers in chinese advanced chronic kidney disease patients. Clin Lab. 2015;61(7):839-43. https://doi.org/10.7754/clin. lab.2015.141101

25. Cejka D, Jäger-Lansky A, Kieweg H, Weber M, Bieglmayer C, Haider DG, et al. Sclerostin serum levels correlate positively with bone mineral density and microarchitecture in haemodialysis patients. Nephrol Dial Transplant. 2012;27(1):226-30. https:// doi.org/10.1093/ndt/gfr270

26. Yamada S, Inaba M, Kurajoh M, Shidara K, Imanishi Y, Ishimura $E$, et al. Utility of serum tartrate-resistant acid phosphatase (TRACP5b) as a bone resorption marker in patients with chronic kidney disease: independence from renal dysfunction. Clin Endocrinol (Oxf) 2008;69(2):189-96. https://doi.org/10.1111/ j.1365-2265.2008.03187.x

27. Bacchetta J, Boutroy S, Guebre-Egziabher F, Juillard L, Drai J, Pelletier $S$, et al. The relationship between adipokines, osteocalcin and bone quality in chronic kidney disease. Nephrol Dial Transplant. 2009;24(10):3120-5. https://doi.org/10.1093/ ndt/gfp262 